\title{
c-Met signalling and endocytosis in clear cell ovarian cancer
}

\author{
G.E.Wood ${ }^{1}$, S.F.Heuss ${ }^{1}$, I.Osagie ${ }^{1}$, M.Lockley², S.Kermorgant ${ }^{1}$ \\ ${ }^{1}$ Centre of Tumour Biology, Barts Cancer Institute, QMUL \\ ${ }^{2}$ Centre of Molecular Oncology, Barts Cancer Institute, QMUL
}

Introduction Clear cell ovarian cancer (CCOC) is a rare subtype of ovarian cancer. Response rate to first line chemotherapy is only $20 \%$. As a result, survival in advanced $\mathrm{CCOC}$ is poor and new therapies are urgently required. Upon binding its ligand, Hepatocyte Growth Factor (HGF), the receptor tyrosine kinase c-Met promotes proliferation, survival and motility. c-Met amplification has been demonstrated in $37 \%$ of CCOC, compared to $2.6 \%$ of epithelial cancers. Recent clinical trials with c-Met inhibitors in ovarian cancer showed response in only $20 \%$ of patients. More comprehensive understanding of c-Met signalling in CCOC is necessary to establish optimal therapeutic strategies. We have previously shown that c-Met transmits oncogenic signalling post-endocytosis and endocytosis inhibition reduces c-Met driven tumourigenesis (Joffre et al., Nat Cell Biol 2011). Consequently, manipulating c-Met endosomal signalling may provide a novel therapeutic approach. c-Met endocytic trafficking has never previously been analysed in CCOC.

\section{c-Met is a target in CCOC}

1- C-Met is expressed and active in CCOC

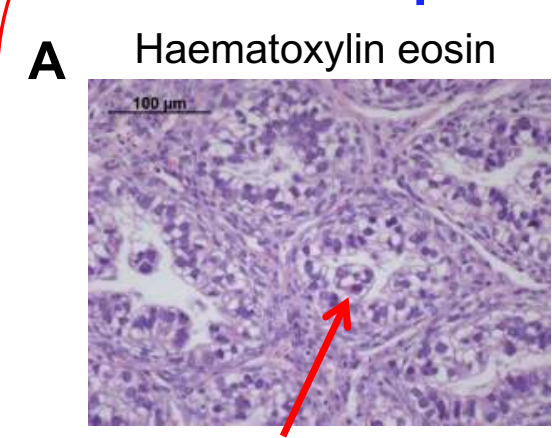

CCOC tumour cells with cytoplasmic clearing

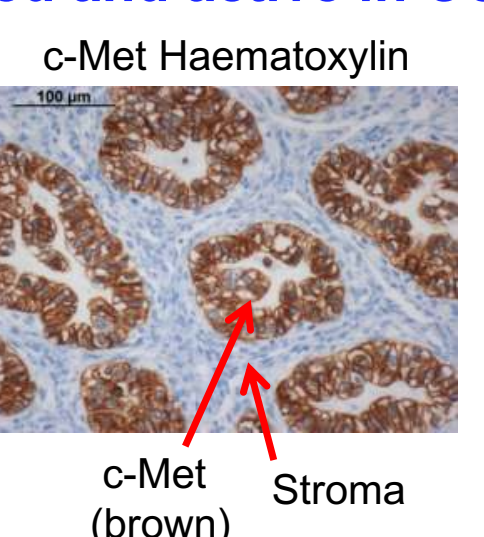

(brown)

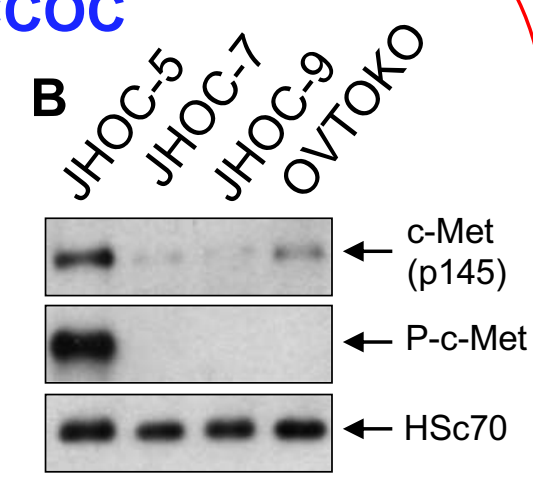

$-\sim-\boldsymbol{H S c 7 0}$

A: Intense c-Met immunostaining (brown) in the malignant epithelial cells of a human CCOC tumour. B: Western blots showing c-Met expression in CCOC cell lines. JHOC-5 cells express constitutively phosphorylated c-Met.

2- c-Met inhibitors reduce c-Met activation and signalling

\begin{tabular}{|c|c|c|c|c|c|c|c|c|c|c|c|c|}
\hline \multirow{3}{*}{$\begin{array}{l}\text { Capmatinib: } \\
\text { HGF(min): }\end{array}$} & \multicolumn{4}{|c|}{ JHOC-7 } & \multicolumn{4}{|c|}{ JHOC-9 } & \multicolumn{4}{|c|}{ OVMANA } \\
\hline & - & - & + & + & - & - & + & + & - & - & + & + \\
\hline & 0 & 15 & 0 & 15 & 0 & 15 & 0 & 15 & 0 & 15 & 0 & 15 \\
\hline & $m$ & - & $=$ & $=$ & $=$ & $=$ & $=$ & $=$ & $=$ & $=$ & $=$ & $=$ \\
\hline c-Met - & 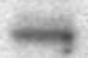 & $\rightarrow$ & $=$ & - & 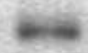 & 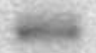 & 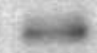 & $\omega$ & - & - & $=$ & - \\
\hline P-c-Met & & $2=$ & $=$ & $=$ & 5 & $x+$ & ent & & & 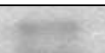 & & \\
\hline & & - & & & & he & & & & $=$ & & \\
\hline
\end{tabular}

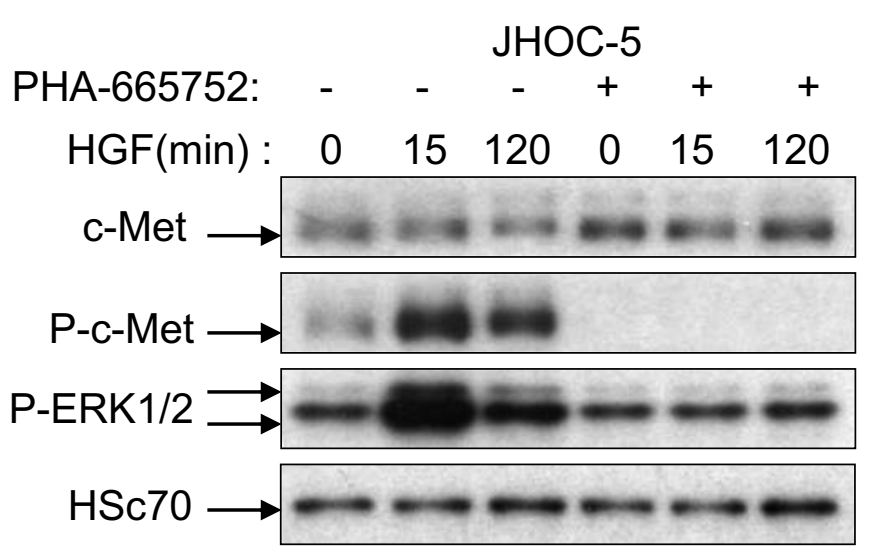

Western blots showing that HGF stimulates c-Met and downstream ERK1/2 phosphorylation.

These responses are inhibited by the c-Met inhibitors Capmatinib (10nM) or PHA-665752 (1 $\mu \mathrm{M})$.

3- Cell survival depends on c-Met expression and activity
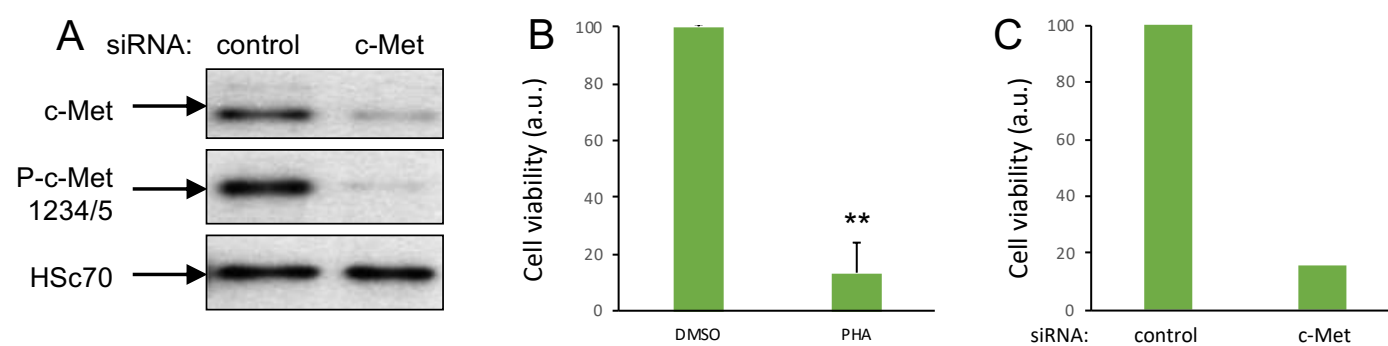

A: Western blot demonstrating effective knockdown of c-Met with siRNA in JHOC-5. B: the c-Met inhibitor PHA-665752 reduced survival $\left(n=3 ;{ }^{* *} p<0.001\right.$, student t-test). C: silencing c-Met reduced survival $(n=2)$.
C-Met signals on endosomes in CCOC

1- HGF stimulation triggers rapid c-Met endocytosis

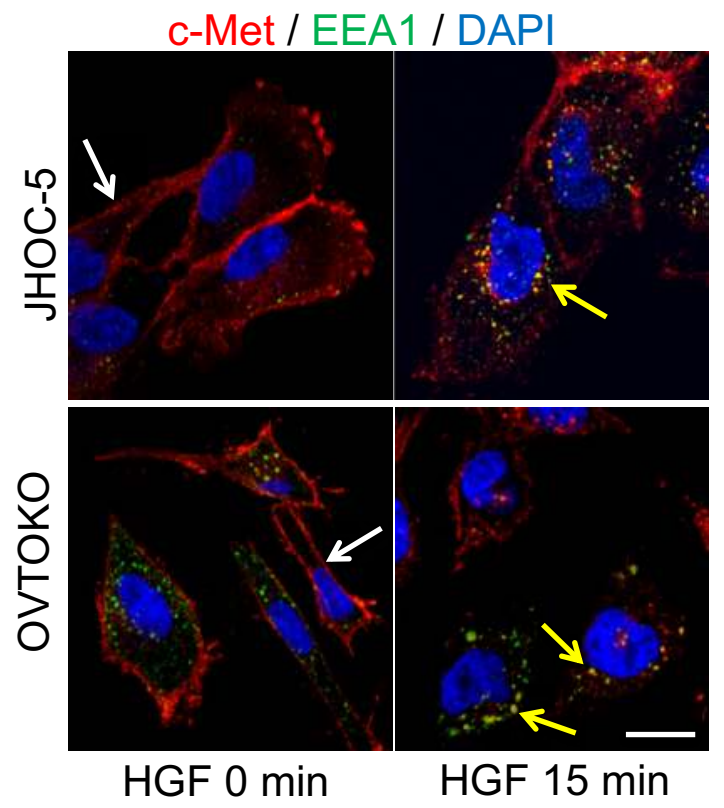

c-Met is localised at the plasma membrane (white arrow) at 0 min, it has endocytosed in vesicles at 15 min of HGF stimulation. Yellow arrows indicate colocalisation between c-Met and EEA1. Scale bar $10 \mu \mathrm{m}$.

$$
\text { HGF } 0 \text { min HGF } 15 \text { min }
$$

2- c-Met endocytosis can be impaired pharmacologically

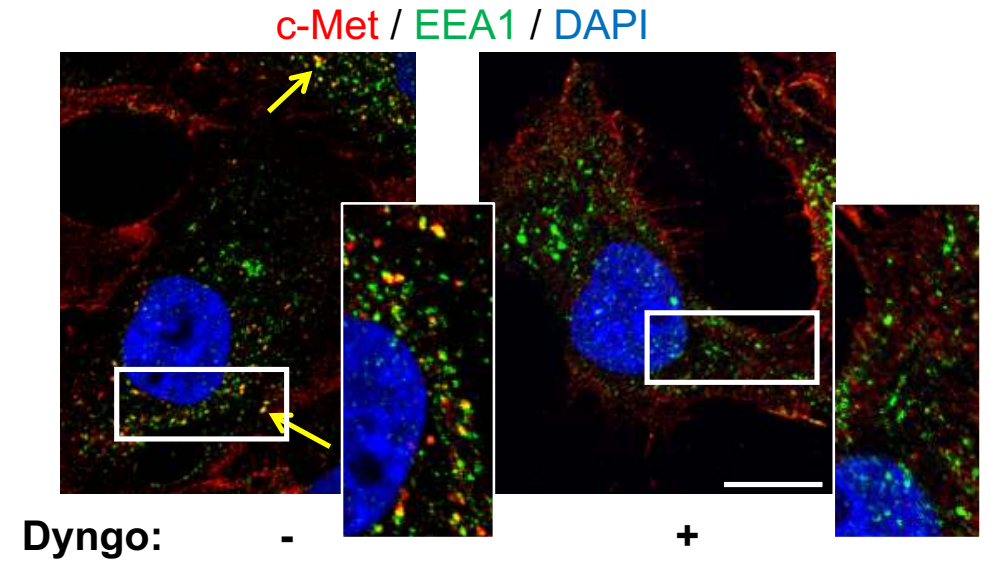

Endocytosis was impaired by pharmacological inhibitor Dyngo in JHOC-5. C-Met colocalisation with EEA1 (yellow arrows) was detected without but not with Dyngo. Scale bar $10 \mu \mathrm{m}$.

3- Endocytosis inhibition reduces c-Met activation and signalling

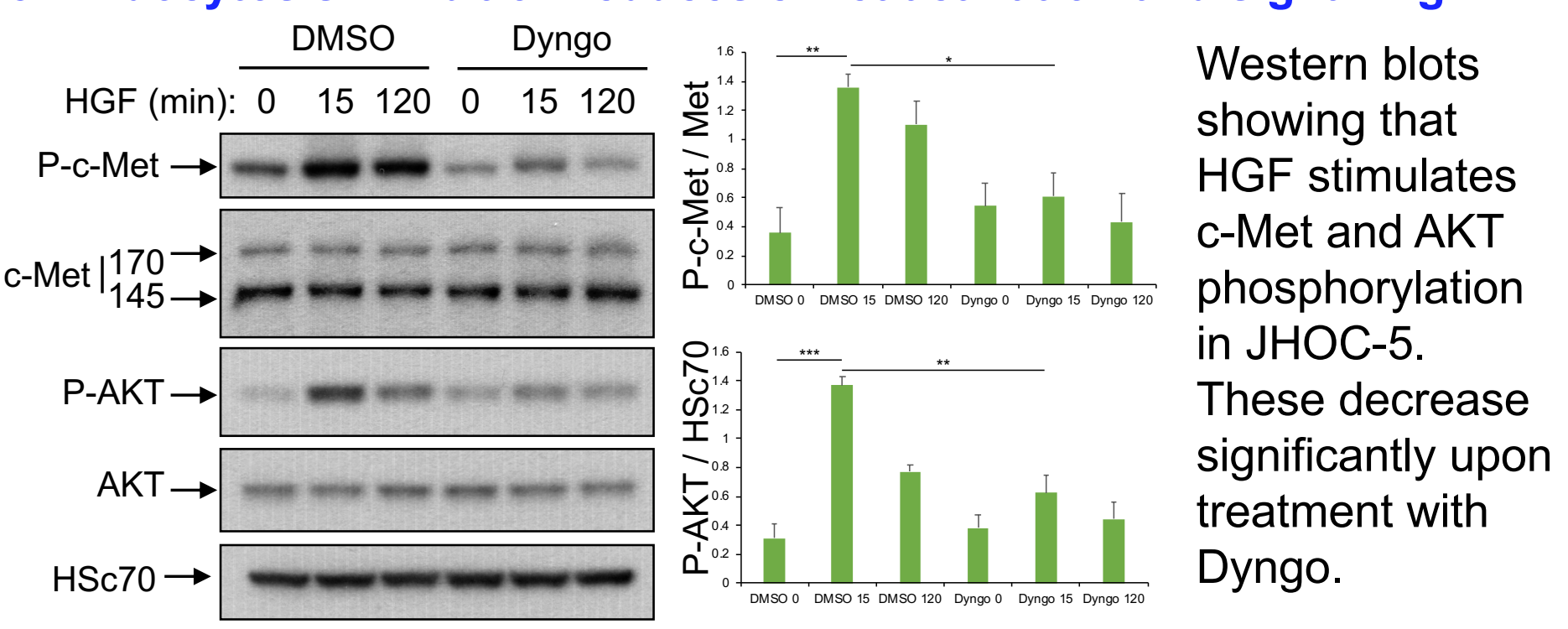

The graphs are densitometry results averaged from $n=3$ independent experiments, student t-test, ${ }^{*} p<0.05,{ }^{* *} p<0.01,{ }^{* * *} p<0.005$.

4- Endocytosis inhibition reduces $\mathrm{C}-$ Met driven cell survival

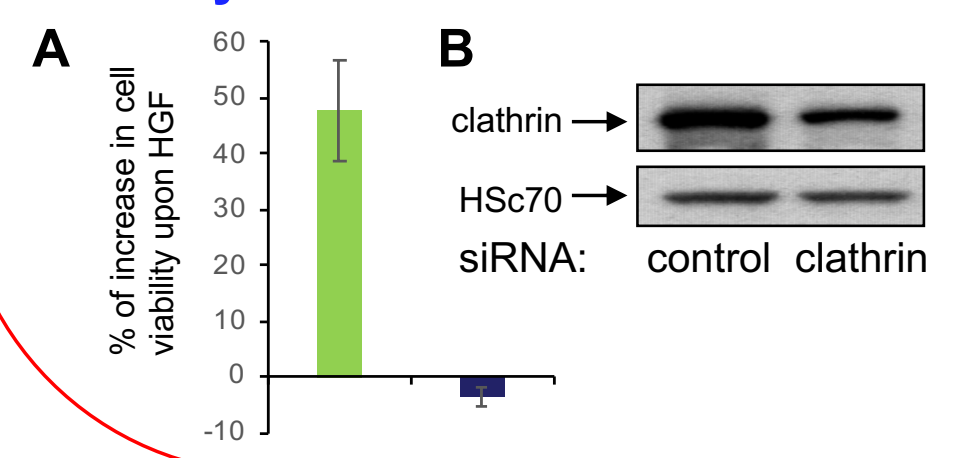

A: Transfection with Clathrin Heavy Chain siRNA impairs endocytosis and reduces cell viability in JHOC-5. B: Western blots for heavy chain of clathrin and HSc70 are shown.

Conclusion This study confirms c-Met as a therapeutic target in CCOC and illustrates, for the first time, that c-Met is endocytosed in CCOC The reduction of c-Met signalling following pharmacological blockade of endocytosis strongly indicates that c-Met needs to internalise to transmit its downstream signalling fully. Further work is ongoing to unravel these pathways and to identify novel treatment approaches. 